\title{
8 Fazit und Schlussfolgerungen
}

\section{Fazit}

In Politik und Medien spricht man aufgrund des demografischen Wandels und der zunehmenden Lebenserwartung meist von einer alternden Gesellschaft, wobei man den zunehmenden Anteil an alten Menschen oftmals als Problem auffasst und dabei die Finanzierung einer zukunftsgerechten Altersvorsorge kontrovers diskutiert. Auch die Wissenschaft beschäftigt sich schon länger mit diesem Thema, indem beispielsweise mögliche Zukunftsszenarien der Pflegebedürftigkeit berechnet werden (vgl. Höpflinger \& Hugentobler 2003). Die Gesundheitsversorgung hat sich in letzter Zeit besonders gewandelt: Während pflegebedürftige alte Menschen im 19. Jahrhundert üblicherweise von der Familie gepflegt wurden und später zu Hause starben, so stirbt heutzutage in vielen Industrieländern die Mehrheit der Menschen in Institutionen wie Spitälern oder Heimen. Und weil dabei besonders die Alters- und Pflegeheimaufenthalte vor dem Tod relativ lang sein können, ist die letzte Lebensphase Vieler zunehmend durch diese Institutionen geprägt.

Übertritte in Institutionen können soziale Sterbeprozesse einleiten und beschleunigen sowie die Partizipationsfähigkeiten der Menschen einschränken, was sich letztlich auch negativ auf die Gesundheit im weiteren Sinne auswirken kann. Es ist davon auszugehen, dass eine Mehrheit der Menschen im Alter so lange wie möglich zu Hause bleiben und dort sterben möchte und analog dazu - wenn es zu einem Übertritt in ein Heim oder Spital kommt - kürzere Aufenthalte in der Regel gegenüber längeren bevorzugt. Daran anschliessend stellt sich die Frage, welche Faktoren für einen verhältnismässig frühen Spital- oder Heimeintritt bzw. für lange Aufenthalte verantwortlich sind und weshalb die Menschen wo sterben. Viele bisherige Studien fanden sowohl bei der Gesundheit als auch bei der Mortalität signifikante Unterschiede: Statushöhere und sozioökonomisch privilegiertere Menschen leben länger und sind gesünder als sozioökonomisch weniger Privilegierte (vgl. Chandola et al. 2007; Huisman et al. 2005; Mielck 2005). In dieser Arbeit interessiert, ob sich solche Unterschiede auch bei Heim- und Spitalaufenthalten sowie unterschiedlichen Sterbeorten feststellen lassen und ob beispielsweise privilegiertere Bevölkerungsgruppen weniger lange Heimaufenthalte vor 
dem Tod haben und seltener in einem Heim sterben. Generell ist dabei besonders bei den Heimaufenthalten und den Heimen als Sterbeort von stärker ausgeprägter vertikaler und horizontaler sozialer Ungleichheit auszugehen als bei Spitalaufenthalten und den Spitälern als Sterbeort.

Die Resultate zeigen summa summarum ein ziemlich eindeutiges Bild: Bei den Heimaufenthalten sind nicht etwa die kränksten bzw. pflegebedürftigsten Personen besonders lange in einem Alters- oder Pflegeheim vor dem Tod, sondern eher solche, die beim Heimeintritt eher weniger pflegebedürftig sind, häufiger alleinlebend sind, keine Kinder haben, und tendenziell einen tieferen soziökonomischen Status haben. Insgesamt spricht einiges für die Kausations- oder Kumulationsthese. Dies würde implizieren, dass der im vorangegangenen Leben eingenommene soziale Status auch im Alter noch gültig ist oder die Statusdifferenzen gar noch anwachsen (vgl. Knesebeck \& Schäfer 2009).

Bei den Spitalaufenthalten sind sozioökonomische oder familiale Faktoren vom Haus- oder Wohneigentum einmal abgesehen - weniger relevant, ob man mehr oder weniger Tage im Spital verbringt im letzten Jahr vor dem Tod. Dafür sind Menschen in der französischsprachigen Schweiz sowie solche, die bereits an Multimorbidität leiden, signifikant länger im Spital als Deutschschweizer bzw. solche, bei denen keine Multimorbidität diagnostiziert wurde. Demnach spricht in diesem Zusammenhang einiges für die These der Altersbedingtheit oder die Destrukturierungsthese, nach denen in erster Linie das Alter und die damit verbundenen psychischen und physischen Veränderungen die soziale Lage prägen und sozioökonomische Faktoren eher zweitrangig sind (vgl. Knesebeck \& Schäfer 2009).

Die Sterbeortanalysen untermauern diese gefundenen Resultate und liefern zusätzliche empirische Evidenz dafür, dass bei den Heimen die Effekte sozialer Ungleichheit stärker sind: Alleinlebend, eher weniger gut ausgebildet, kein Wohneigentum sowie keine Kinder sind alles Merkmale einer Bevölkerungsgruppe, die mit einer höheren Wahrscheinlichkeit in einem Heim als zu Hause stirbt als Menschen in einer Beziehung, eher gut gebildet, Wohn- oder Hauseigentümer sowie mehrfache Eltern von Kindern. Demgegenüber finden sich bei den Spitälern kaum eindeutige Hinweise auf soziale Einflussfaktoren. Bei den Todesfällen nach kurzen wie langen Aufenthalten - beispielsweise bedingt durch medizinische Notfälle - spricht insgesamt mehr für medizinische Determinanten, was letztlich nicht weiter überraschend ist. Besonders auffällig sind dagegen die Unterschiede zwischen den Sprachregionen: In der Westschweiz wie auch im Tessin sterben signifikant mehr Personen in Institutionen als in der Deutschschweiz, 
besonders in Spitälern. Insgesamt spricht mehr empirische Evidenz für die Kausations- oder Kumulationsthese.

Es fällt auf, dass die gefundenen Resultate bei den Spital- und Heimaufenthalten sich bei den Sterbeortanalysen grösstenteils bestätigen: Die potenziell vulnerable Bevölkerungsgruppe hat längere Heimaufenthalte vor dem Tod, stirbt verhältnismässig häufig dort, ist vorwiegend weiblich und häufiger alleinstehend, stirbt verhältnismässig häufig an Demenz oder an einem Schlaganfall, ist durchschnittlich tiefer gebildet und häufiger ohne Wohneigentum sowie häufiger multimorbid und zum Zeitpunkt des Heimeintrittes in den Alltagsaktivitäten weniger eingeschränkt. Vermutlich haben viele Personen dieser angesprochenen Gruppe bei einer eintretenden Pflegebedürftigkeit weniger Ressourcen als privilegiertere Gruppen, um auf die neue Lebenssituation zu reagieren. Beispielswiese könnten Besitzer eines Hauses einfacher altersgerechte Umbauten vornehmen als Mieter, Verheiratete können vom Ehepartner gepflegt werden und Paare mit Kindern von ihren eigenen Kindern. Je weniger Optionen man diesbezüglich hat, desto höher scheint die Wahrscheinlichkeit eines Heimeintrittes. Eine Studie über familiale Pflegearrangements (vgl. Haberkern 2009) fand heraus, dass Personen ohne ausreichend finanzielle Mittel mit höherer Wahrscheinlichkeit keine oder weniger familiale Pflege erhalten, was die Vermutung bestätigen könnte, dass weniger Pflegeoptionen - sowohl familial als auch finanziell - eher zu einem Heimeintritt führen, selbst wenn die Pflegebedürftigkeit noch verhältnismässig gering ist.

Bei Spitalaufenthalten vor dem Tod existieren weniger Alternativen, denn medizinische Behandlungen können in der Regel nur von Experten durchgeführt werden. Auch hier zeigen sich - wie bei den Heimaufenthalten - auffällige Parallelen zwischen den Aufenthalten und dem Sterbeort: Der Einfluss von sozialen Faktoren ist insgesamt nicht eindeutig, vielmehr finden sich dafür Unterschiede zwischen den Sprachregionen, was sich sowohl bei der Aufenthaltsdauer als auch beim Sterbeort deutlich zeigt.

In dieser Arbeit wurde generell davon ausgegangen, dass eine Mehrheit der Betroffenen kürzere Aufenthalte in Institutionen in der Regel bevorzugt und möglichst zu Hause sterben möchte. Diese Wünsche sind offenbar nicht für alle Bevölkerungsschichten gleich realistisch, denn schliesslich zeigen sich, wie diese Arbeit darlegt, soziale Ungleichheiten in der Versorgung am Lebensende. Aufgrund theoretischer Überlegungen sind zudem hinter den Gesundheitsindikatoren wie beispielsweise der Multimorbidität (vgl. Barnett et al. 2012) weitere Einflüsse von sozialen Faktoren denkbar, die bereits zu einer ungleichen Verteilung der Gesundheit geführt haben, noch bevor die Versorgung am Lebensende 
überhaupt relevant wird. Demnach könnte die kumulierte soziale Ungleichheit vielleicht noch stärker ausgeprägt sein als unsere Resultate anhand der Versorgung zeigen. Das nicht signifikante Ergebnis der Dichte von Heimbetten nach Region bei den Heimaufenthaltsdaueranalysen lässt sich vielleicht folgendermassen deuten: Der Wohlfahrtsstaat ermöglicht auf der einen Seite zwar, der Bevölkerung bei Bedarf einen Heim- oder Spitalplatz zur Verfügung zu stellen, schafft es aber - wie ein Vergleich mit den anderen Resultaten der Modelle zeigt - auf der anderen Seite nicht, sozioökonomische Ungleichheit im Laufe des höheren Lebensalters durch Umverteilung komplett auszugleichen.

Die in dieser Arbeit verwendeten administrativen Daten haben viel Potenzial und eignen sich mit einem innovativen und kreativen Forschungsdesign durchaus für soziologische Fragestellungen. Aufgrund von theoretischen Überlegungen wurde entschieden, drei separate Erklärungsmodelle mit jeweils einer anderen abhängigen Variable und einer anderen Studienpopulationen zu rechnen. Dabei passen die Resultate der drei Teile inhaltlich gut zusammen und sind nicht nur in sich konsistent, sondern über alle drei Modelle hinweg, was im Nachhinein sowohl die Wahl der Indikatoren als auch das teilweise explorative Vorgehen rechtfertigt.

Abschliessend kann man sich fragen, inwieweit die Befunde auch auf andere Länder übertragbar sind. Wie eine Metaanalyse über unterschiedliche Sterbeorte (vgl. Broad et al. 2013) aufzeigt, gibt es innerhalb der Industrieländer grosse Unterschiede, besonders was die Anteile der Verstorbenen in Heimen betrifft. Dies könnte implizieren, dass besonders die vorliegenden Resultate zu den Heimen schwierig auf andere Länder anzuwenden sind. Vor allem dürften die unterschiedlichen Arten von Wohlfahrtsstaaten einen entsprechenden Einfluss darauf haben, ob sich die Menschen beispielsweise eine Pflege in Institutionen überhaupt leisten können oder ob und wie stark die familiale Pflege verbreitet ist (vgl. Esping-Andersen 1990; Haberkern 2009). Und bei den Spitälern dürfte es neben den kulturellen Vorstellungen des Lebensendes auch eine Rolle spielen, wie gut ausgebaut die medizinische Versorgung insgesamt ist. Beispielsweise variieren die Gesundheitsausgaben massiv zwischen den einzelnen Ländern (vgl. Kocher 2009). Nichtsdestotrotz ist denkbar, dass die Mechanismen und Effekte innerhalb der jeweiligen Personengruppe von beispielsweise Heimbewohnern, die durch diverse vorhergegangene Selektionsprozesse eine verhältnismässig homogene Gruppe darstellen, mit den in dieser Arbeit verwendeten Studienpopulationen verglichen werden können. 


\section{Gesundheitsversorgung und Geschlecht}

Nach diesem allgemeinen Fazit wird nun auf vier besonders auffällige Befunde im Detail eingegangen. Als erstes ist dabei die Rolle der Geschlechter zu nennen. Aufgrund des Vorgehens mit geschlechtsspezifischen Modellen sind jedoch direkte Vergleiche zwischen Männern und Frauen nicht zulässig, dafür sind Auffälligkeiten, die in beiden Stichproben auftreten, inhaltlich miteinander vergleichbar. Obwohl wir von elementaren Unterschieden zwischen den Geschlechtern ausgegangen sind, zeigen sich letztlich innerhalb der Geschlechter vergleichbare Effekte. In den Alters- und Pflegeheimen gibt es zwar absolut gesehen viel mehr Frauen als Männer, die durchschnittlich auch älter sterben, was angesichts der höheren Lebenserwartung nicht weiter überrascht und sich auch mit unseren theoretischen Überlegungen deckt. Aber offensichtlich sind für diese beiden Geschlechtergruppen letztlich mehrheitlich die gleichen Faktoren relevant, und dies gleich bei allen drei Modellen. Eine mögliche Erklärung dafür könnten die bereits vor dem Heim- oder Spitaleintritt stattgefundenen Selektionsprozesse sein, welche letztlich diese Bevölkerungsgruppe in vielen Merkmalen homogenisieren, wodurch das Geschlecht an Bedeutung verliert. Gerade bei den Männern, die neben einer geringeren Lebenserwartung in den Heimen die Minderheit stellen sowie viel kürzere durchschnittliche Aufenthaltsdauern als Frauen haben, könnte der Effekt des selektiven Überlebens einiges erklären (vgl. Markides \& Machalek 1984): Diejenigen, die einerseits alt werden und andererseits in ein Heim eintreten, stellen eine Personengruppe unter den Männern dar, die sich von den Männern in jüngeren Lebensjahren unterscheidet und sich in vielen Parametern den Frauen der vergleichbaren Altersgruppe annähert. Auch bei den Spitalaufenthalten könnte dieser Selektionsmechanismus gewirkt haben, wobei dort die Geschlechterunterschiede geringer ausgeprägt sind.

\section{Gesundheitsversorgung und Wohneigentum}

Weiterhin auffällig sind die konsistenten Resultate in Bezug auf Wohn- oder Hauseigentum. Im Gegensatz zur Bildung ist dieser Indikator sowohl bei den Männern als auch bei den Frauen durchwegs signifikant: Haus- oder Wohnungseigentümer sind kürzer im Spital, haben kürzere Heimaufenthalte und sterben mit geringerer Wahrscheinlichkeit im Spital oder Heim gegenüber Nichteigentümern. Wenn man die beiden Indikatoren vertikaler sozialer Ungleichheit - also Wohn- oder Hauseigentum und höchster Bildungsabschluss - vergleicht, fällt zudem auf, dass das Bildungsniveau üblicherweise vor dem Wohn- oder Hausei- 
gentum erworben wird und dass letztlich ein biografisch aus der Sicht des Lebensendes näher liegender Statusindikator eine konsistentere Wirkung ausübt.

Etwas überraschend ist das bei der Spitalaufenthaltsdauer gefundene gegensätzliche Resultat von Bildung und Wohn- oder Hauseigentum bei den Männern: Ein höheres Bildungsniveau erhöht die Anzahl Spitaltage signifikant gegenüber dem mittleren Bildungsniveau, während Wohn- oder Hauseigentum die Anzahl Tage gegenüber den Nichteigentümern signifikant verkürzt. Diese Tatsache führt zu einer etwas unklarer Ausprägung sozialer Ungleichheit bei Spitalaufenthalten: Einerseits ist denkbar, dass privilegiertere Bevölkerungsgruppen gesünder sind und deshalb weniger Spitalbehandlungen benötigen; andererseits könnten diese Bevölkerungsgruppen für Spitäler, wenn sie sich beispielswiese aufgrund besserer Finanzen eher Zusatzversicherungen leisten, so attraktiv sein, dass sie gerne über längere Zeit als notwendig im Spital behalten werden und vielleicht auch mehr Behandlungen erhalten und dadurch längere Aufenthalte hätten. Letztlich ist und bleibt dies anhand unserer Resultate spekulativ, es zeigt aber definitiv die insgesamt weniger eindeutige Bedeutung des sozioökonomischen Status bei den Spitalaufenthalten gegenüber den Heimaufenthalten auf.

\section{Gesundheitsversorgung und Krankheiten}

Als nächstes finden sich einige Auffälligkeiten bei den Krankheiten. Diagnostizierte Multimorbidität erhöht für beide Geschlechter signifikant die Spitalaufenthaltsdauer und bei Männern auch die Heimaufenthaltsdauer. Einen sehr starken Effekt findet man erwartungsgemäss für Frauen und Männer bei den Hospitalisationen im letzten Lebensjahr während des Heimaufenthaltes. Demnach gehen Spitaleintritte mit einer verhältnismässig deutlich verschlechternden Gesundheit einher, was den Heimaufenthalt stark verkürzt, weil die betroffenen Personen vermutlich bald darauf sterben.

Aufschluss über die Krankheiten in der letzten Lebensphase geben auch die Todesursachen. Aufgrund der Aufenthaltsdauer in Spitälern - und in geringerem Ausmass in Heimen - ist aber nicht klar, ob einige davon schneller zum Tod führen als andere oder ob man einfach weniger Spital- oder Heimaufenthalte benötigt. Anhand einiger Resultate zeigen sich dennoch Tendenzen, die man interpretieren kann. Wenn man beispielsweise an Demenz verstirbt, so ist die Wahrscheinlichkeit für Männer und Frauen hoch, dass man einerseits in einem Heim stirbt und andererseits auch lange im Heim lebt, bevor man stirbt. Gleichzeitig ist die Wahrscheinlichkeit von längeren oder mehreren Spitalaufenthalten im letzten Lebensjahr erhöht. Vermutlich hängt dieser Effekt mit dem schleichenden Krank- 
heitsverlauf und der im Verhältnis relativ hohen Lebenserwartung von Demenz zusammen, der im Vergleich zu anderen Krankheiten wie Krebs weniger schnell zum Tod führt, aber die Betroffenen dafür längere Zeit von Pflege abhängig macht, die vorwiegend in Heimen oder auch zu Hause stattfindet. Aufgrund der weiter zunehmenden Lebenserwartung dürften demenzielle Erkrankungen in den nächsten Jahren noch weiter zunehmen (vgl. Höpflinger \& Hugentobler 2003), was im Endeffekt vielleicht zu mehr und noch längeren Heimaufenthalten von Betroffenen führen kann.

\section{Gesundheitsversorgung und Sprachregionen}

Nicht zuletzt sind auch die Unterschiede zwischen den Landesteilen auffällig. Etwas vorsichtig sollte man die Resultate für den Kanton Tessin im Vergleich zu den anderen beiden, deutlich grösseren Landesteilen, interpretieren. Letztlich zeigt sich der Hauptunterschied aber generell zwischen der lateinischen und deutschsprachigen Schweiz: In der lateinischen Schweiz sind die Menschen vor dem Tod signifikant länger im Spital und sterben mit einer signifikant höheren Wahrscheinlichkeit in einem Spital oder Heim als zu Hause im Vergleich mit der Deutschschweiz. Insgesamt spricht anhand dieser Resultate einiges für kulturell unterschiedliche Vorstellungen bezüglich der Versorgung am Lebensende zwischen den Landesteilen oder zumindest zwischen den Sprachregionen, denn die Unterschiede sind einerseits ziemlich stark ausgeprägt und andererseits konsistent. Es scheint nachvollziehbar, dass wenn die betroffenen Personen im letzten Lebensjahr mehr Zeit in einem Spital verbringen, auch mit einer höheren Wahrscheinlichkeit dort sterben.

Aufschlussreich ist vielleicht ein Vergleich mit den jeweils gleichsprachigen Nachbarländern, wo sich tatsächlich interessante Parallelen erkennen lassen: In Frankreich sterben beispielswiese - gemessen an allen Todesfällen - fast $60 \%$ der Menschen in Spitälern, rund 30\% zu Hause und nur rund 10\% in Alters- und Pflegeheimen (Broad et al. 2013). In Deutschland wurde bis anhin keine landesweite Studie über unterschiedliche Sterbeorte durchgeführt, immerhin gibt es eine Studie über Verstorbene in Rheinland-Pfalz, die wiederum alle Verstorbenen untersuchte: Rund 40\% sterben zu Hause, weitere rund 40\% in Spitälern, knappe $15 \%$ in Alters- und Pflegeheimen und weitere gute 5\% anderswo wie beispielsweise in Palliativeinrichtungen (Escobar Pinzon et al. 2013). Auch wenn diese Zahlen nur bedingt untereinander vergleichbar sind und im Gegensatz zu den Ergebnissen aus dieser Arbeit nicht auf die ältere Bevölkerung beschränkt sind, so sterben offensichtlich in der Deutschschweiz und in Deutschland gegenüber 
der französischsprachigen Schweiz und Frankreich mehr Menschen zu Hause als in Institutionen. Zudem sind die Alters- und Pflegeheime im deutschsprachigen Raum als Sterbeorte etwas verbreiteter als im französischsprachigen Raum. Die Ausgaben für spitalexterne Pflegeleistungen hingegen sind in der französischsprachigen Schweiz tendenziell etwas höher als in der Deutschschweiz (Obsan 2011).

\section{Sozialpolitische Implikationen}

Was für Konsequenzen können aus dieser Arbeit für Gesellschaft und Politik gezogen werden? Die alternde Gesellschaft stellt in jedem Fall eine grosse Herausforderung dar. Falls die Pflegebedürftigkeit und die medizinische Versorgung bzw. die medizinischen Behandlungen am Lebensende in Zukunft weiter steigen werden, wovon man aufgrund der gegenwärtigen Entwicklung ausgehen kann (vgl. Höpflinger \& Hugentobler 2003), ist der Wohlfahrtstaat gleich doppelt gefordert: Einerseits dürften die Kosten der Versorgung vor dem Lebensendes weiter ansteigen, was die Frage einer gerechten Finanzierung aufwirft, und andererseits könnte die soziale Ungleichheit der Gesundheitsversorgung noch zunehmen, wenn beispielsweise die Alters- und Pflegeheime weiter an Bedeutung gewinnen und die in dieser Arbeit gefundenen Ungleichheiten für eine noch grössere Bevölkerungsgruppe relevant werden.

Wie könnte man den Herausforderungen begegnen? Generell wünschenswert ist eine Versorgung am Lebensende, die in diesem für alle Betroffenen sehr schwierigen Kontext von Krankheiten, Pflegebedürftigkeit, physischem und psychischem Abbau, verminderten Partizipationsfähigkeiten sowie physischem, psychischem und sozialem Sterben die Wünsche und Bedürfnisse der betroffenen alten Menschen sowie deren Angehörigen möglichst berücksichtigt. Eine diesbezügliche Optimierung könnte nebenbei auch Kosten einsparen: Wenn beispielsweise weniger privilegierte Bevölkerungsgruppen mehr Möglichkeiten bei einer eintretenden Pflegebedürftigkeit offen stehen, beispielsweise durch ausgebaute spitalexterne Pflege, so könnte dies bei den Betroffenen vielleicht zu einem späteren Heimeintritt führen und dadurch die teuren langen Heimaufenthalte verkürzen, was gleichzeitig ja auch dem Wunsch vieler Betroffenen nachkäme, möglichst lang in ihrer vertrauten Umgebung leben zu können.

Bei den Heimaufenthalten stellt sich die Frage, ob man aus Sicht des Wohlfahrtsstaates den vulnerablen Bevölkerungsgruppen mehr Alternativen zu einem verhältnismässig frühen Heimeintritt bei geringer Pflegebedürftigkeit zur Verfügung stellen soll. Beispielsweise könnte ein Ausbau der ambulanten, spitalexter- 
nen Pflege dafür sorgen, dass leicht pflegebedürftige Personen länger zuhause leben können. Hierbei müsste man vielleicht die bisherigen Finanzierungsmodelle überdenken, um auch weniger privilegierten Bevölkerungsgruppen den $\mathrm{Zu}$ gang zu erleichtern. Damit einhergehend sollte man dies mit einem Ausbau von altersgerechten Wohnungen unterstützen, etwa indem man Fahrstühle in ältere Häuser einbaut und die Wohnungen dadurch auch für Personen bewohnbar macht, die in ihrer Bewegungsfähigkeit eingeschränkt sind. Auch innovative Ideen könnten vielleicht zur Problemlösung beitragen: Alterswohngemeinschaften mit einem Mix aus familialer und professioneller Pflege helfen vielleicht, leicht pflegebedürftigen Menschen einen mehr oder weniger autonomen Alltag länger aufrechtzuerhalten. Und in Städten könnten vielleicht Studenten günstig bei älteren, leicht pflegebedürftigen Menschen wohnen und als Gegenleistung Unterstützung im Alltag leisten.

Daran anschliessend stellt sich die Frage, was man mit präventiven Massnahmen noch erreichen könnte, um den alten Menschen eine möglichst lange autonome und behinderungsfreie Zeit zu ermöglichen. Demenz beispielsweise ist eine Krankheit mit degenerativem Verlauf und verhältnismässig langen Heim- und Spitalaufenthalten. Wenn man die Demenzerkrankungsraten reduzieren könnte, hätte dies neben einer besseren Gesundheit mit einer hohen Wahrscheinlichkeit durchschnittlich kürzere Aufenthalte in Institutionen zur Folge. Neben der genetischen Disposition gibt es einige bekannte Risikofaktoren wie Depression, Bluthochdruck, physische Inaktivität, Übergewicht, Diabetes, erhöhte Cholesterinkonzentration sowie Rauchen, die das Demenzrisiko im Alter signifikant erhöhen (Deckers et al. 2015). Unter diesen Indikatoren finden sich einige Risikofaktoren wie etwa Rauchen oder Übergewicht, die man bereits als Risikofaktor für weitere Krankheiten kennt und die bereits Gegenstand von einigen bisherigen Präventionskampagnen waren. Dennoch könnte sich noch mehr gezielte Prävention auszahlen, denn wenn die Lebenserwartung noch weiter steigt, nimmt vermutlich je nach Entwicklungsszenario - auch die Prävalenz von Demenz weiter zu, wodurch dieses Thema in Zukunft vielleicht noch wichtiger wird (vgl. Höpflinger \& Hugentobler 2003). Bisherige aus dem Alltag bekannte Gesundheitstipps wie viel Bewegung, nicht zu rauchen oder eine gesunde Ernährung wirken sich also auch in Bezug auf ein geringeres Demenzrisiko aus, was im Endeffekt, aufgrund des geringeren Risikos langer Heimaufenthalte, die Lebensqualität am Lebensende positiv beeinflussen kann. Hierbei könnte man die Prävention besonders auf vulnerable Bevölkerungsgruppen ausrichten, denn bisherige Studien zeigen, dass beispielsweise Rauchen und Übergewicht in weniger privilegierten Bevölke- 
rungsgruppen verbreiteter sind als in privilegierteren Bevölkerungsgruppen (vgl. Mielck 2005; Østbye et al. 2002).

Bei den Spitälern scheint die wohlfahrtsstaatliche Absicherung zu funktionieren, ein Problem dürfte eher der grosse Anteil von Todesfällen darstellen. Aus Sicht der vorliegenden Resultate scheint es angebracht zu konstatieren, dass sich Spitäler vermehrt mit dieser Herausforderung auseinandersetzten sollten und vielleicht vermehrt akzeptieren, dass eine kurative Behandlung kurz vor dem Tod nicht immer die sinnvollste ist. Diese Herausforderung anzugehen, dürfte naturgemäss schwierig sein, denn neben den medizinischen Aspekten spielen auch ethische, moralische und rechtliche Argumente eine wichtige Rolle. Die Debatten um aktive und passive Sterbehilfen zeigen beispielsweise, wie empfindlich die Gesellschaft bei Fragen über das Lebensende reagiert. Die zunehmend auch in Spitälern integrierten Palliativpflegestationen sind sicherlich eine weithin akzeptierte und passende Antwort auf diese Herausforderungen und kommen dem gestiegenen Bedürfnis Vieler nach mehr Selbstbestimmung am Lebensende entgegen. Besonders bei degenerativen und letalen chronischen Krankheiten ohne Aussicht auf Heilung scheinen - wenn sie denn im Spital längere Zeit behandelt werden - spezialisierte Palliativpflegeabteilungen Sinn zu machen.

\footnotetext{
Open Access Dieses Kapitel wird unter der Creative Commons Namensnennung-Nicht kommerziell 4.0 International Lizenz (http://creativecommons.org/ licenses/by-nc/4.0/deed.de) veröffentlicht, welche für nicht kommerzielle Zwecke die Nutzung, Vervielfältigung, Bearbeitung, Verbreitung und Wiedergabe in jeglichem Medium und Format erlaubt, sofern Sie den/die ursprünglichen Autor(en) und die Quelle ordnungsgemäß nennen, einen Link zur Creative Commons Lizenz beifügen und angeben, ob Änderungen vorgenommen wurden.

Etwaige Abbildungen oder sonstiges Drittmaterial unterliegen ebenfalls der genannten Creative Commons Lizenz, sofern sich aus der Abbildungslegende oder der Quellreferenz nichts anderes ergibt. Sofern solches Drittmaterial nicht unter der genannten Creative Commons Lizenz steht, ist eine Vervielfältigung, Bearbeitung oder öffentliche Wiedergabe nur mit vorheriger Zustimmung des betreffenden Rechteinhabers oder auf der Grundlage einschlägiger gesetzlicher Erlaubnisvorschriften zulässig.
} 